\title{
Differences in false recollection according to the cognitive reserve of healthy older people
}

\section{Alfonso Pitarque, Juan Meléndez, Alicia Sales, Teresa Mayordomo, Joaquín Escudero \& Salvador Algarabel}

To cite this article: Alfonso Pitarque, Juan Meléndez, Alicia Sales, Teresa Mayordomo, Joaquín Escudero \& Salvador Algarabel (2016) Differences in false recollection according to the cognitive reserve of healthy older people, Aging, Neuropsychology, and Cognition, 23:5, 625-637, DOI: 10.1080/13825585.2016.1146221

To link to this article: http://dx.doi.org/10.1080/13825585.2016.1146221

册 Published online: 16 Mar 2016.

Submit your article to this journal $\asymp$

Џ Article views: 39

Q View related articles $\sqsubset$

View Crossmark data $\nearrow$ 


\title{
Differences in false recollection according to the cognitive reserve of healthy older people
}

\author{
Alfonso Pitarque (10) ${ }^{a}$, Juan Meléndez (1) ${ }^{a}$, Alicia Sales (10) ${ }^{a}$, Teresa Mayordomo ${ }^{a}$, \\ Joaquín Escudero ${ }^{b}$ and Salvador Algarabel $^{a}$
}

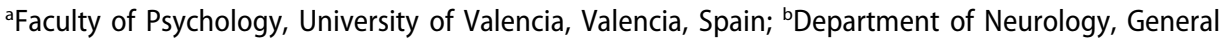
Hospital, Valencia, Spain

\begin{abstract}
We present an associative recognition experiment comparing three samples of healthy people (young people, older people with high cognitive reserve $[\mathrm{HCR}]$, and older people with low cognitive reserve [LCR], with each sample consisting of 40 people), manipulating stimuli repetition during the study phase. The results show significant differences among the three samples in their overall performance. However, these differences are not due to a different use of familiarity, but rather due to a different way of using recollection: although there are no differences in the hit rates between the HRC and LRC samples, the LCR group makes significantly more recollective false alarms than the HCR group. Moreover, repetition provokes an increase in the recollective false alarms in the LCR group, but this does not occur in the group of young people or in the HCR group. These findings are explained in terms of recollection-based monitoring errors and seem to provide support for the cognitive reserve hypothesis.
\end{abstract}

\section{ARTICLE HISTORY}

Received 8 September 2015 Accepted 19 January 2016

\section{KEYWORDS}

False recollection; associative recognition; aging; cognitive reserve; memory

\section{Introduction}

The main objective of our study is, using an associative recognition paradigm, to analyze whether the cognitive reserve level of healthy older people affects their rates of false recollections (i.e., false alarms [FAs] of recollective type, which also called phantom recollections; Brainerd, Wright, Reyna, \& Mojardin, 2001), and how this is affected by the repetition of the pairs of stimuli to be studied.

It is well known that age and cognitive impairment lead to a decrease in the performance on different cognitive functions, especially memory. However, not all types of memory decline with age in the same way. For example, there is a broad consensus in the literature that the so-called explicit or episodic memory deteriorates considerably, whereas the implicit or semantic memory seems to decline less or even remain intact (e.g., Dew \& Giovanello, 2010; Fleischman \& Gabrieli, 1998; Light \& Singh, 1987). Similarly, the so-called dual-process recognition models (e.g., Yonelinas, 2002) postulate that two processes intervene in recognition: recollection, which is conceived of as a conscious process involving a threshold-type qualitative retrieval of episodic 
traces and tends to decline with age; and familiarity, which is considered an automatic retrieval process based on the quantitative estimation of the strength of the memory trace and tends to remain intact with age. In contrast to the dual models, the singleprocess or global strength theories (e.g., Dunn, 2004) propose that information retrieval from the memory is based only on a quantitative estimation of the trace strength: what dual models call familiarity would refer to weak memories, while recollection would refer to strong memories.

In addition, there are clear individual differences in the way this cognitive decline appears. For example, the cognitive reserve hypothesis (Stern, 2009) establishes that lifelong experiences, including educational and occupational achievement and leisure activities in later life, are associated with a slower rate of memory decline in normal aging and a reduced risk of developing dementia. People with high cognitive reserve (HCR) may somehow be able to compensate for cognitive decline by implementing cognitive strategies or, at a more functional level, by using more complex neural connections. Thereby, this hypothesis proposes that healthy older people with HCR would make better use of their cognitive strategies to delay cognitive decline than older people with low cognitive reserve (LCR). If this is true, in a recognition experiment, it would be expected, for example, that both samples differ in the use of recall-to-reject strategy to reduce their FA rates (Rotello \& Heit, 2000) which involve rejecting a nonpresented item because one can consciously recollect the presentation of its instantiating target (e.g., Gallo, Sullivan, Daffner, Schacter, \& Budson, 2004).

There is also a large body of research showing that false recognition (and false memories) increases with age in healthy people (e.g., Abe et al., 2011; Dodson, Bawa, \& Slotnick, 2007; Hildebrandt, Haldenwanger, \& Eling, 2009; McCabe, Roediger, McDaniel, \& Balota, 2009; Norman \& Schacter, 1997). Associative recognition is an optimal paradigm for studying false recognition in older people (Cohn \& Moscovitch, 2007), because it has been established that aging causes more impairment in recognition memory for associations than for components (e.g., Boywitt, Kuhlmann, \& Meiser, 2012; Kilb \& NavehBenjamin, 2011; Old \& Naveh-Benjamin, 2008; Rhodes, Castel, \& Jacoby, 2008). In this regard, the associative-binding deficit hypothesis (Old \& Naveh-Benjamin, 2008) proposes that older adults' episodic memory impairments are partially due to a reduced ability to encode and retrieve associated/bound units of information (e.g., Buchler, Faunce, Light, Gottfredson, \& Reder, 2011; Fandakova, Shing, \& Lindenberger, 2013). In a recent study by our laboratory using an associative recognition task (Pitarque, Sales, Meléndez, \& Algarabel, 2015), we found that repetition increased false recollections in older people, but not in younger people. This result was interpreted as indicating that young people (but not elderly people) were able to recollect the original associate of one of the items in order to reject the rearranged lures, using repetition to improve this recall-to-reject monitoring strategy (Rotello \& Heit, 2000). However, elderly people responded to rearranged pairs mainly based on the high activation (increased by repetition) of each item, which led them to commit false recollections (Gallo et al., 2004). If this explanation is correct, and based on the cognitive reserve hypothesis, healthy older people with HCR would be expected to use their monitoring strategies to reduce their rates of false recollections through practice better than older people with LCR. Given the theoretical relevance of the cognitive reserve hypothesis (Stern, 2009), and that there are experimental inconsistencies related to it (see, e.g., Angel, Fay, Bouazzaoui, Baudouin, \& 
Isingrini, 2010, or Valenzuela \& Sachdev, 2006, supporting it, but Zahodne et al., 2011, refuting it), we now propose to analyze whether this expected pattern of results is maintained when comparing a sample of LCR older people and a sample of HCR older people, or whether the HCR group behaves like the group of young people (i.e., no increase in false recollections due to repetitions), which would support the cognitive reserve hypothesis.

More specifically, we propose an associative recognition experiment (recognition of faces associated with everyday scenery, with remember-know [RK] judgments; e.g., Kilb \& Naveh-Benjamin, 2011, exp. 2) in which half of the pairs are repeated during the study task, which allows us to analyze whether older people with HCR make fewer false recollections than older people with LCR. A control group of healthy young people is also included to serve as a baseline for comparing the recognition of the samples of older people with HCR and LCR. With regard to true recognition, we hypothesize that the three samples differ in their overall performance by their different use of recollection, but not familiarity, as the so-called dual-process recognition models postulate (e.g., Yonelinas, 2002). With regard to false recognition, and according to the cognitive reserve hypothesis, we hypothesize that older people with HCR should use repetition better than the people with LCR to improve their recall-to-reject monitoring strategy to reduce their rates of false recollections.

\section{Methods}

\section{Participants}

Participants were 40 young adults (undergraduates at the University of Valencia; 13 men, 27 women, mean age $=22.05$ years, SD $=2.97$, range $18-29$ years old) and 80 older adults (recruited from various senior citizen centers in the city of Valencia; 22 men, 58 women, mean age $=68.31$ years, $S D=6.96$, range 60-90 years old). All participants reported being in good physical and mental health. In this regard, the mean on the MiniMental State Examination (MMSE) for the older adults was 28.86 (SD =1.19), showing no memory impairment.

The HCR and LCR older groups were created based on the individual cognitive reserve score for each older participant established according to the guidelines recommended in previous studies (e.g., Solé-Padullés et al., 2009; Stern et al., 2005). Individual performance was measured on three variables (see Table 1): (a) the WAIS vocabulary scale (range 1-19); (b) education level ( 1 = illiteracy; no formal studies, 2 = primary studies, 3 = secondary studies, 4 = university studies) plus occupation ( 1 = non-qualified manual, 2 = qualified manual, 3 = qualified non-manual or technician, 4 = professional; university degree required, $5=$ manager or director; university degree required), yielding a composite score ranging from 2 to 9; and (c) leisure activities (reading, writing, sports, daily walking, social activities, etc.), ranging from 0 to 14 . Then, a principal component factor analysis was conducted on these three measures, and a single-factor solution explained $72.3 \%$ of the variance. The final individual cognitive reserve score (range: 6.60-25.50) was obtained by multiplying each original individual variable by its factor loading (e.g., Solé-Padullés et al., 2009). Based on this continuous variable, and to draw comparisons with young adults, we formed two groups of healthy older people, LCR and 
Table 1. Means (and SE) of the participant characteristics.

\begin{tabular}{lcccc}
\hline & $\begin{array}{c}\text { A. YOUNG } \\
(n=40)\end{array}$ & $\begin{array}{c}\text { B. HCR OLDER } \\
(n=40)\end{array}$ & $\begin{array}{c}\text { C. LCR OLDER } \\
(n=40)\end{array}$ & $\begin{array}{c}\text { Significant differences } \\
(p<.05)\end{array}$ \\
\hline Gender (men/women) & $13 / 27$ & $12 / 28$ & $10 / 30$ & \\
Age & $22.05(0.47)$ & $67.00(0.78)$ & $69.63(1.32)$ & $\mathrm{A}<(\mathrm{B}=\mathrm{C})$ \\
Mini-Mental (MMSE) & & $29.25(0.13)$ & $28.48(0.22)$ & $\mathrm{B}>\mathrm{C}$ \\
Cognitive reserve: & & $20.52(0.32)$ & $12.61(0.46)$ & $\mathrm{B}>\mathrm{C}$ \\
$\quad$ WAlS vocabulary & $9.03(0.41)$ & $12.50(0.34)$ & $10.23(0.31)$ & $\mathrm{B}>(\mathrm{A}=\mathrm{C})$ \\
Education level (scale 1-4) & $4.00(0.00)$ & $3.58(0.10)$ & $2.03(0.14)$ & $\mathrm{A}>\mathrm{B}>\mathrm{C}$ \\
Occupation level (scale 1-5) & & $3.53(0.15)$ & $1.53(0.13)$ & $\mathrm{B}>\mathrm{C}$ \\
Leisure activities (scale 0-14) & & $8.95(0.28)$ & $4.78(0.42)$ & $\mathrm{B}>\mathrm{C}$ \\
\hline
\end{tabular}

HCR (matched for gender and age), dichotomizing from the median (=17.15): the cognitive reserve mean of the LCR group $(n=40)$ was 12.61 (SD $=2.88$ ), whereas the mean of the HCR group $(n=40)$ was $20.52(S D=2.04)$.

\section{Materials}

The same materials were used as in Pitarque et al. (2015, pictures pairs). They consisted of 64 ID-card sized color photographs ( $145 \times 160$ pixels) of anonymous faces ( 16 of older men, 16 of older women, 16 of young men, and 16 of young women) and 64 color photographs of unknown everyday scenery $(800 \times 600$ pixels; e.g., Kilb \& NavehBenjamin, 2011). With these photographs, we then created four different lists of 64 pairs of photographs each, putting a randomly chosen face in the center of a randomly chosen scene. These four lists of images were counter-balanced between subjects, and later analyses of the hits and FAs confirmed that there were no significant differences between them.

\section{Procedure}

The associative recognition task consisted of a study phase with 64 pairs of stimuli and a recognition task with 60 pairs, with a 5-minute break between them. In the study phase, 60 pairs of stimuli (plus 2 pairs of distractors at the beginning and at the end, not tested later to avoid primacy and recency effects) were presented randomly at the center of a computer screen for 2.5 seconds each (with a 1-second interval between them). Participants were instructed to pay attention to the two pictures in each pair. In the study task, 20 pairs were presented once (later making up the non-repeated intact and non-repeated rearranged recognition conditions), and 20 pairs were presented twice (later making up the repeated intact and the repeated rearranged recognition conditions).

In the recognition phase (self-paced), 60 pairs of stimuli were presented randomly at the center of a computer screen. The participants had to decide whether or not the two pictures in each pair had appeared together in the study task, choosing one of these four response options: (a) yes, because I remember some details; (b) yes, because I know they went together, but not the details; (c) no, both stimuli appeared before, but they are rearranged; (d) no, neither stimulus appeared before; they are new. That is, participants only had to respond "yes" to intact pairs, as commonly occurs in associative recognition experiments. Of the 60 pairs of stimuli presented on the recognition task, 
10 corresponded to the non-repeated intact condition, 10 corresponded to the repeated intact condition, 10 corresponded to the non-repeated rearranged condition (randomly re-matching the stimuli in a different order from the one studied), 10 corresponded to the repeated rearranged condition (randomly re-matching the stimuli in a different order), and 20 corresponded to the new condition. Prior to performing the first recognition task, and following strict RK instructions (Koen \& Yonelinas, 2014), the differences between "remembering" and "knowing" were explained to participants (see Kilb \& Naveh-Benjamin, 2011, exp. 2), emphasizing that a remember response should only be given if they could communicate a retrieved detail to the experimenter if asked, whereas a know response should be given if they believed the pair had previously been studied, but they were unable to retrieve any specific details. A practice recognition task was performed to make sure all the subjects understood the instructions.

\section{Results}

\section{General recognition performance (total "yes" judgments)}

With regard to discriminability data ( $d$ '; see Table 2 ), a mixed ANOVA of 3 groups $\times 2$ repetition conditions ${ }^{1}$ showed that the main effects of both variables were significant, groups $\left(F_{2,117}=69.40, p<.0001, \eta_{p}^{2}=.54\right)$ and repetition conditions $\left(F_{1,117}=17.96\right.$, $p<.0001, \eta_{p}^{2}=.13$ ), indicating that repeated stimuli led to better discrimination than non-repeated stimuli (means $=2.00$ and 1.45, respectively). Post-hoc Bonferroni $t$-tests comparing the means of the three groups showed as significant $(p<.05)$ all the differences between them (means $=2.71,1.61$, and 0.86 , for the young, HCR, and LCR groups, respectively), indicating that young people performed better than the HCR group, and the latter, in turn, performed better than LCR group. The interaction between the two variables was also significant $\left(F_{2,117}=5.86, p<.01, \eta_{p}^{2}=.09\right)$. Regarding the analysis of this significant interaction (Table 2), post-hoc Bonferroni $t$-tests showed that the discriminability improved with repetition in both the young and HCR groups (young group's means: 2.21 and 3.20 for non-repeated and repeated conditions, respectively, $t_{39}=4.22, p<.0001$; HCR group's means: 1.26 and 1.96, respectively, $t_{39}=2.94, p<.01$ ), but not in the LCR group (means: 0.88 and 0.83 for non-repeated and repeated conditions, respectively), which seems to provide support for the cognitive reserve hypothesis.

With regard to hits (see Table 2), a mixed ANOVA of 3 groups $\times 2$ repetition conditions showed that the main effects of both variables were significant, groups $\left(F_{2,117}=7.76, p=.001, \eta_{p}^{2}=.12\right)$ and repetition conditions $\left(F_{1,117}=69.70, p<.0001\right.$, $\left.\eta_{p}^{2}=.37\right)$, indicating that repeated stimuli led to more hits than non-repeated stimuli (means $=0.83$ and 0.66 , respectively), as would be expected. Post-hoc Bonferroni $t$-tests comparing the means of the three groups showed significant differences between the mean of the young group (0.82) and the means of the HCR and LCR groups ( 0.70 and 0.71 , respectively, with the difference not being significant). The interaction between the two variables was not significant.

Regarding FAs (see Table 2), a mixed ANOVA of 3 groups $\times 3$ repetition conditions (non-repeated, repeated, and new pairs) showed that the main effects of both variables were significant, groups $\left(F_{2,117}=57.18, p<.0001, \eta_{p}^{2}=.49\right)$ and repetition conditions $\left(F_{2,234}=108.45, p<.0001, \eta_{p}^{2}=.48\right)$. Post-hoc Bonferroni $t$-tests comparing the means 
Table 2. Means (and SE) of hits, false alarms, and discriminability index ( $d^{\prime}$ ) on the associative recognition task as a function of groups and repetition conditions (Non-rep: non-repeated pairs; Rep: repeated pairs; New: new pairs) .

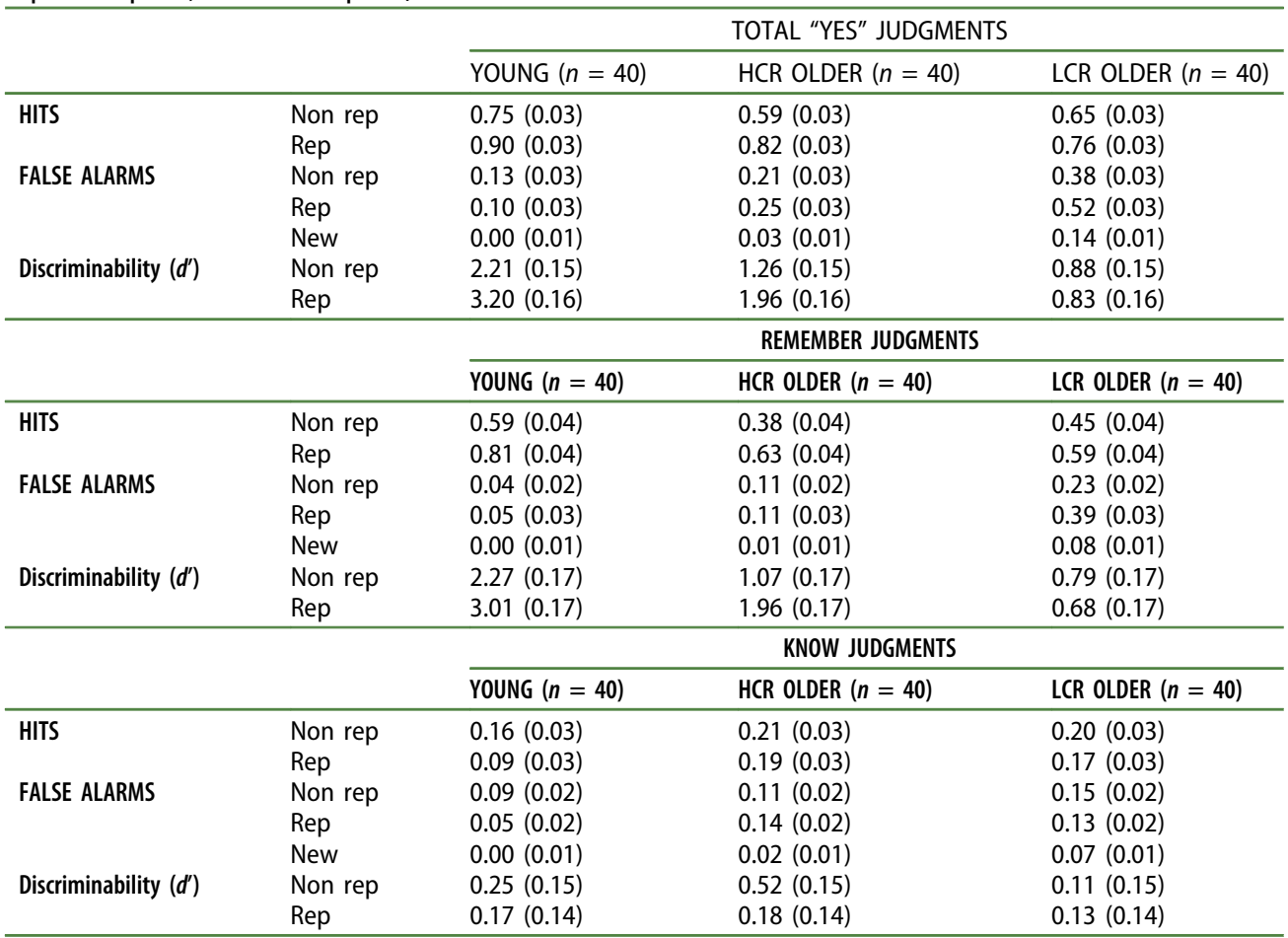

of the three groups showed that all the differences between them were significant $(0.08$, 0.17 , and 0.35 , for the young, HCR, and LCR groups, respectively). Post-hoc Bonferroni $t$-tests comparing the means of the three repetition conditions showed that all the differences were significant $(0.24,0.29$, and 0.06 , for non-repeated, repeated, and new pairs, respectively). Finally, the interaction between the two variables was also significant $\left(F_{4,234}=13.04, p<.0001, \eta_{p}^{2}=.18\right)$. Regarding the analysis of this significant interaction (Table 2), simple effects analysis showed that repetition conditions (non-repeated, repeated, and new pairs) affected the three groups $\left(F_{2,78}=16.29, p<.0001\right.$, $\eta_{p}^{2}=.30 ; F_{2,78}=32.24, p<.0001, \eta_{p}^{2}=.45 ; F_{2,78}=68.76, p<.0001, \eta_{p}^{2}=.64$, for the young, HCR, and LCR groups, respectively). Post-hoc Bonferroni $t$-tests comparing the means of the three repetition conditions in each group (Table 2) showed that in the LCR group, all the differences were significant (means: $0.38,0.52$, and 0.14 for nonrepeated, repeated, and new pairs, respectively), indicating that repeated pairs gave rise to more FAs than non-repeated pairs. However, in the HCR group, the difference between non-repeated and repeated conditions was not significant (means: 0.21, 0.25, and 0.03 for non-repeated, repeated, and new pairs, respectively), as also occurred in the young group (means: $0.13,0.10$, and 0.00 , respectively), that is, repetition increased FAs in the LCR group, but not in the HCR and young groups. Repetition has been shown to increase FAs to rearranged lures in older adults in a number of other studies (e.g., Fandakova et al., 2013; Gallo et al., 2004; Light, Patterson, Chung, \& Healy, 2004; Pitarque 
et al., 2015; Rhodes et al., 2008). We will later analyze whether this increase in the LCR group is due to false recollections or familiarity.

Overall, the results show significant differences among the three samples in their performance, but the HCR group discriminated better than the LCR group because they committed fewer FA than the LCR group (with similar hit rates in both samples). Moreover, the learning capacity through practice is preserved in the older HCR group (using it to reduce their FAs), just as in the young people, but it is seriously limited in the older LCR group.

\section{Remember judgments}

With regard to discriminability data $\left(d^{\prime}\right)$ on remember $(\mathrm{R})$ judgments (Table 2), an ANOVA of 3 groups $\times 2$ repetition conditions showed similar results to those found for the $d^{\prime}$ of total "yes" responses; that is, the main effect of the variable "groups" was significant $\left(F_{2,117}=53.67, p<.0001, \eta_{p}^{2}=.48\right)$. Post-hoc Bonferroni $t$-tests comparing the means of the three groups showed significant differences among the three groups (means $=2.67,1.52$, and 0.74 , for the young, HCR, and LCR groups, respectively). The main effect of repetition conditions was also significant $\left(F_{1,117}=20.42, p<.0001\right.$, $\eta_{p}^{2}=.15$ ), indicating that repeated stimuli yielded better discrimination through $R$ judgments than non-repeated stimuli (means $=1.91$ and 1.38 , respectively), and the interaction between these two variables was also significant $\left(F_{2,77}=7.55, p=.001\right.$, $\eta_{p}^{2}=.11$ ). Regarding the analysis of this significant interaction (Table 2), post-hoc Bonferroni $t$-tests showed that both the young group and the HCR group improved their performance on $\mathrm{R}$ judgments with repetition (young group's means: 2.27 and 3.01 , for non-repeated and repeated pairs, respectively, $t_{39}=3.72, p=.001$; HCR group's means: 1.07 and 1.96, respectively, $\left.t_{39}=3.90, p<.0001\right)$, but the LCR group did not (means: 0.79 and 0.68 , for non-repeated and repeated pairs, respectively).

The mixed $3 \times 2$ ANOVA of hits on $\mathrm{R}$ judgments (Table 2 ) also showed a similar pattern of results to what was found for total hits; that is, the main effects of both variables were significant, groups $\left(F_{2,117}=10.36, p<.0001, \eta_{p}^{2}=.15\right)$ and repetition conditions $\left(F_{1,117}=99.21, p<.0001, \eta_{p}^{2}=.46\right)$, but their interaction was not, indicating again that repeated stimuli gave rise to more hits on $R$ judgments (mean $=0.68$ ) than non-repeated stimuli (mean $=0.48$ ). Post-hoc Bonferroni $t$-tests comparing the means of the three groups showed significant differences between the mean of the young group (0.70) and the means of the HCR and LCR groups (0.51 and 0.52, respectively, with the difference not being significant), indicating that both older samples might show an associative-binding deficit (Old \& Naveh-Benjamin, 2008).

Finally, regarding FA on $R$ judgments (see Table 2), a mixed ANOVA of 3 groups $\times$ 3 repetition conditions (non-repeated, repeated, and new pairs) showed that the main effects of both variables were significant, groups $\left(F_{2,117}=39.37, p<.0001\right.$, $\left.\eta_{p}^{2}=.40\right)$ and repetition conditions $\left(F_{2,234}=68.92, p<.0001, \eta_{p}^{2}=.37\right)$. Post-hoc Bonferroni $t$-tests comparing the means of the three groups showed significant differences between the mean of the LCR group (0.23) and the means of the HCR and young groups $(0.08$ and 0.03 , respectively, with the difference not being significant). Post-hoc Bonferroni $t$-tests comparing the means of the three repetition conditions showed that all the differences were significant $(0.12,0.18$, and 0.03 , for 
non-repeated, repeated, and new pairs, respectively). Finally, the interaction between the two variables was also significant $\left(F_{4,234}=18.86, p<.0001, \eta_{p}^{2}=.24\right)$. Regarding the analysis of this significant interaction (Table 2), simple effects analysis showed that repetition conditions (non-repeated, repeated, and new pairs) affected all the three groups $\left(F_{2,78}=8.85, p<.0001, \eta_{p}^{2}=.19 ; F_{2,78}=13.54, p<.0001, \eta_{p}^{2}=.26 ; F_{2}\right.$, $78=51.41, p<.0001, \eta_{p}^{2}=.57$, for the young, HCR, and LCR groups, respectively). Post-hoc Bonferroni $t$-tests comparing the means of the three repetition conditions in each group (Table 2) showed that in the LCR group, all the differences were significant (means: 0.23, 0.39, and 0.08 for non-repeated, repeated, and new pairs, respectively), indicating that repeated pairs led to more $F A$ on $R$ judgments than non-repeated pairs. However, for the HCR group, the difference between the nonrepeated and repeated conditions was not significant (means: $0.11,0.11$, and 0.01 for non-repeated, repeated, and new pairs, respectively), and the same thing occurred in the young group (means: 0.04, 0.05, and 0.00, respectively), indicating in both groups that repetition did not increase the FA rates on $\mathrm{R}$ judgments. In other words, repetition increased FA on $\mathrm{R}$ judgments only in the LCR group, but not in the HCR and young groups. These results show that HCR seems to decrease false recollection (comparing HCR vs LCR groups). For some authors, false recollection could be considered an early indicator of cognitive impairment (e.g., Hildebrandt et al., 2009; McCabe et al., 2009).

Overall, the results found on R judgments mimic the results found on total "yes" judgments, showing again that the LCR group discriminated worse through $\mathrm{R}$ judgments than the HCR group because they committed more FA on R judgments (but the same hits) than the HCR group. Moreover, the HCR group (as in young people) improved its performance on $\mathrm{R}$ judgments through practice, whereas the LCR group did not.

\section{Know judgments}

With regard to discriminability data (d') on know (K) judgments (Table 2), a mixed ANOVA of 3 groups $\times 2$ repetition conditions revealed that the main effects and interaction between the two variables were not significant.

Regarding hits on $\mathrm{K}$ judgments, the mixed $3 \times 2$ ANOVA revealed that the main effects and interaction of the two variables were non-significant. Some authors (Koen \& Yonelinas, 2014; Yonelinas, 2002) argue that $\mathrm{K}$ judgments are not good estimators of true familiarity, proposing new methods to estimate it. For example, the independence method (Koen \& Yonelinas, 2014; Yonelinas, 2002) proposes the result yielded by the formula (hits on $\mathrm{K}$ judgments)/( 1 - hits on $\mathrm{R}$ judgments) as a good estimator of true familiarity. Using it as dependent variable in a new ANOVA of 3 groups $\times 2$ repetition conditions, the results revealed that the main effects and the interaction of the two variables were again non-significant. These results show that familiarity seems to be stable throughout a healthy life cycle (e.g., Algarabel et al., 2009; Koen \& Yonelinas, 2014; Yonelinas, 2002).

Finally, regarding FA made on K judgments (Table 2), a mixed ANOVA of 3 groups $\times 3$ repetition conditions (non-repeated, repeated, and new pairs) showed that the main effects of both variables were significant, groups $\left(F_{2,117}=9.51, p<.0001, \eta_{p}^{2}=.14\right)$ and repetition conditions $\left(F_{2,234}=33.90, p<.0001, \eta_{p}^{2}=.23\right)$. Post-hoc Bonferroni $t$-tests 
comparing the means of the three groups showed significant differences between the mean of the young group (0.05) and the LCR and HCR groups $(0.12$ and 0.09 , respectively, with the difference not being significant), indicating that young people use familiarity better than elderly people to correctly reject new information. Post-hoc Bonferroni $t$-tests comparing the means of the three repetition conditions showed significant differences between the new pairs (mean: 0.03) and the non-repeated and repeated pairs (means: 0.11 and 0.11 , respectively, with the difference not being significant). However, the interaction between the two variables was not significant, which, in relation to the main objective of our study, means that the increase in total FA produced by the repetitions in the LCR group (but not in the HCR or young groups) is due to the incorrect use of recollection, and not familiarity, with implications that we discuss later.

\section{Discussion}

In general, our results provide support for the cognitive reserve hypothesis by showing that the HCR group performed significantly better than the LCR group on the associative recognition task. However, this difference is not due to a different use of familiarity in the HRC and LRC groups, as both samples use familiarity in a similar way in recognition (as they get similar rates of hits, FA, and $d^{\prime}$ on $\mathrm{K}$ judgments). The difference in the performance of the HRC and LRC groups must lie in the different ways the two samples use recollection, both correctly (hits) and, especially, incorrectly (FA).

Regarding correct recognition based on $\mathrm{R}$ judgments, our data show that, although there are no significant differences in the hit rates on $\mathrm{R}$ judgments between the HRC and LRC samples, the HRC sample benefits from the stimulus repetition in a similar way to the young people. Both samples improve significantly with practice, although, logically, starting at different basal levels. However, the LCR sample does not show any improvement with stimulus repetition, suggesting that they can have an associative-binding deficit (Old \& Naveh-Benjamin, 2008).

Regarding FA based on $\mathrm{R}$ judgments (or "phantom" recollections), our data reveal significant differences among the three samples, showing that the false recognition increases with age (e.g., Buchler et al., 2011; Kilb \& Naveh-Benjamin, 2011; McCabe et al., 2009) and, as a novel finding, that cognitive reserve seems to decrease false recollection (comparing HCR vs LCR groups). Likewise, our data show that repetition increases false recognition (e.g., Buchler et al., 2011; Gallo et al., 2004; Pitarque et al., 2015; Rhodes et al., 2008). However, as an original contribution, we have shown that this increase in false recognition produced by stimulus repetition is not due to an incorrect use of familiarity, but rather due to an increase in false recollections that only occurs in the LCR group, but not in the HCR group or in young people. Thus, this result would confirm the idea that young people and older people with HCR correctly use the recall-to-reject mechanism to reduce false recollection on rearranged pairs (e.g., Gallo et al., 2004), because they would be capable of rejecting a reordered pair by remembering that the face presented in this pair was studied in association with another scenario (or by remembering that the scenario presented was associated with another face).

In summary, our results show that the HCR group shows similar behavior to the group of young people (even though they start from different basal levels), which can be 
interpreted as an indicator of good cognitive functioning. However, and as a novelty, we have shown that the LCR group shows significantly higher rates of false recollection than the other two groups (which could indicate early cognitive decline; Hildebrandt et al., 2009; McCabe et al., 2009), and that their FA on R judgments increase with stimulus repetition, showing that these subjects have an impaired ability to use recall to reduce false recognition (e.g., Gallo et al., 2004). What seems to occur in the rearranged pairs is that both young people and elderly HCR people (but not elderly LCR people) are able to recollect the original associate of one of the items in order to reject the rearranged lures, using repetition to improve this recall-to-reject monitoring strategy. However, elderly LCR people show a monitoring deficit and respond to rearranged pairs mainly based on the high activation of each item, which is increased by repetition (Gallo et al., 2004).

However, we must be cautious in our conclusions about the role of cognitive reserve because our samples may differ on other variables apart from the cognitive reserve. For example, in our data (older people, $n=80$ ) cognitive reserve correlated significantly with FA on $\mathrm{R}$ judgments (made on non-repeated, repeated, and new stimuli, $r=-.37, r=-.62$, and $r=-.50$, respectively, all $p<.001$ ), but it did not correlate with hits, which seem to show the protective role of cognitive reserve on false recollections. The same pattern of significant correlations was found in the variables that were part of our definition of cognitive reserve (see also Solé-Padullés et al., 2009): WAIS vocabulary, education level, occupation level, and leisure activities. However, age also correlated with FA (made on non-repeated and repeated pairs, $r=.24$ and $r=.28$, respectively, $p<.05$, although our samples were matched on age), but not with hits. Finally, the Mini-Mental scores neither correlated with hits nor with FAs on R judgments (probably neither the LCR nor the HCR samples showed cognitive impairment). However, none of the former variables significantly correlated with either hits or FA on $\mathrm{K}$ judgments, which seems to suggest that aging affects recollection errors, but not familiarity (e.g., McCabe et al., 2009). Therefore, our results can only be considered cumulative evidence for the cognitive reserve hypothesis, in accordance with other publications (see, e.g., Angel et al., 2010; Gordon, Soldan, Thomas, \& Stern, 2013; Valenzuela \& Sachdev, 2006).

Our results reinforce the idea that false recollection in the LCR group can be the result of a decline in both binding (at encoding) and monitoring mechanisms on retrieval (e.g., Fandakova et al., 2013). When processing stimuli that share perceptual characteristics, the LCR participants seem to first encode the stimuli through weaker episodic traces than young people, mistakenly combining features from studied events due to their binding deficits (Old \& Naveh-Benjamin, 2008). Second, their retrieval can produce misrecollections or source monitoring errors, produced by their inability to use recall to reduce false recognition (Abe et al., 2011; Gallo et al., 2004).

Our results seem to provide support for the dual-process recognition models (e.g., Yonelinas, 2002) in the sense that familiarity does not seem to be affected by age, while the recollection does. Our results could also be explained by other dual models related to the false memories literature as, for example, by the activation/monitoring framework (Roediger, Watson, McDermott, \& Gallo, 2001) which explains both true recognition and false recognition in terms of two components: an automatic activation of the studied words that spreads to non-studied related lures (which could lead the LCR group to recollect a false association in a rearranged pair), and a recollection-based monitoring process involving conscious decision-making (which could lead both young and HCR 
samples to reduce their false recognitions). Similarly, our results are consistent with the familiarity plus corroboration or content-borrowing account (Lampinen, Meier, Arnal, \& Leding, 2005) which suggests that when an item lure is highly activated by the presentation of several related items (either perceptually or conceptually), the subject searches for evidence that this item lure was presented, sometimes borrowing false contextual details from the actual items.

In summary, our experiment shows that stimulus repetition increases recollection-type false recognition, but only in older people with LCR, while it does not affect young people or older people with HCR (who are capable of correcting by using monitoring strategies to counteract the effect of the high activation, increased by repetition, of the items). Thus, our results seem to provide support for the cognitive reserve hypothesis (Stern, 2009).

\section{Note}

1. As HCR and LCR groups differed significantly in their Mini-Mental scores (MMSE, but neither group showed cognitive impairment; see Table 1), data were also analyzed by mixed ANCOVA, taking the Mini-Mental scores as covariate. As the ANCOVA and ANOVA results were similar, we only present the latter here.

\section{Disclosure statement}

No potential conflict of interest was reported by the authors.

\section{Funding}

This work was supported by the Dirección General de Investigación Científica y Técnica (proyectos I+D+i) of the Spanish Ministerio de Economía y Competitividad [grant number PSI2013-43533-R].

\section{ORCID}

Alfonso Pitarque (10) http://orcid.org/0000-0003-3367-2607

Juan Meléndez (1) http://orcid.org/0000-0003-3367-2607

Alicia Sales (1) http://orcid.org/0000-0002-7070-8130

\section{References}

Abe, N., Fujii, T., Nishio, Y., lizuka, O., Kanno, S., Kikuchi, H., .. Mori, E. (2011). False item recognition in patients with Alzheimer's disease. Neuropsychologia, 49, 1897-1902. doi:10.1016/j. neuropsychologia.2011.03.015

Algarabel, S., Escudero, J., Mazón, J., Pitarque, A., Fuentes, M., Peset, V., \& Lacruz, L. (2009). Familiaritybased recognition in the young, healthy elderly, mild cognitive impaired and Alzheimer's patients. Neuropsychologia, 47, 2056-2064. doi:10.1016/j.neuropsychologia.2009.03.016

Angel, L., Fay, S., Bouazzaoui, B., Baudouin, A., \& Isingrini, M. (2010). Protective role of educational level on episodic memory aging: An event-related potential study. Brain \& Cognition, 74, 312323. doi:10.1016/j.bandc.2010.08.012

Boywitt, C. D., Kuhlmann, B. G., \& Meiser, T. (2012). The role of source memory in older adults' recollective experience. Psychology and Aging, 27, 484-497. doi:10.1037/a0024729 
Brainerd, C. J., Wright, R., Reyna, V. F., \& Mojardin, A. H. (2001). Conjoint recognition and phantom recollection. Journal of Experimental Psychology: Learning, Memory, and Cognition, 27, 307-327.

Buchler, N. G., Faunce, P., Light, L. L., Gottfredson, N., \& Reder, L. M. (2011). Effects of repetition on associative recognition in young and older adults: Item and associative strengthening. Psychology and Aging, 26, 111-126. doi:10.1037/a0020816

Cohn, M., \& Moscovitch, M. (2007). Dissociating measures of associative memory: Evidence and theoretical implications. Journal of Memory and Language, 57, 437-454. doi:10.1016/j. jml.2007.06.006

Dew, I. T. Z., \& Giovanello, K. S. (2010). Differential age effects for implicit and explicit conceptual associative memory. Psychology and Aging, 25, 911-921. doi:10.1037/a0019940

Dodson, C. S., Bawa, S., \& Slotnick, S. D. (2007). Aging, source memory, and misrecollections. Journal of Experimental Psychology: Learning, Memory, and Cognition, 33, 169-181.doi:10.1037/ 0278-7393.33.1.169

Dunn, J. C. (2004). Remember-know: A matter of confidence. Psychological Review, 111, 524-542. doi:10.1037/0033-295X.111.2.524

Fandakova, Y., Shing, Y. L., \& Lindenberger, U. (2013). Differences in binding and monitoring mechanisms contribute to lifespan age differences in false memory. Developmental Psychology, 49, 1822-1832. doi:10.1037/a0031361

Fleischman, D. A., \& Gabrieli, J. (1998). Repetition priming in normal aging and Alzheimer s disease: A review of findings and theories. Psychology and Aging, 13, 88-119. doi:10.1037/08827974.13.1.88

Gallo, D. A., Sullivan, A. L., Daffner, K. R., Schacter, D. L., \& Budson, A. E. (2004). Associative recognition in Alzheimer's disease: Evidence for impaired recall-to-reject. Neuropsychology, 18, 556-563. doi:10.1037/0894-4105.18.3.556

Gordon, L. T., Soldan, A., Thomas, A. K., \& Stern, Y. (2013). Effect of repetition lag on priming of unfamiliar visual objects in young and older adults. Psychology and Aging, 28, 219-231. doi:10.1037/a0030929

Hildebrandt, H., Haldenwanger, A., \& Eling, P. (2009). False recognition helps to distinguish patients with Alzheimer's disease and amnestic $\mathrm{MCl}$ from patients with other kinds of dementia. Dementia and Geriatric Cognitive Disorders, 28, 159-167. doi:10.1159/000235643

Kilb, A., \& Naveh-Benjamin, M. (2011). The effects of pure pair repetition on younger and older adults' associative memory. Journal of Experimental Psychology: Learning, Memory, and Cognition, 37, 706-719.

Koen, J. D., \& Yonelinas, A. P. (2014). The effects of healthy aging, amnestic mild cognitive impairment, and Alzheimer's disease on recollection and familiarity: A meta-analytic review. Neuropsychology Review, 24, 332-354. doi:10.1007/s11065-014-9266-5

Lampinen, J. M., Meier, C. R., Arnal, J. D., \& Leding, J. K. (2005). Compelling untruths: Content borrowing and vivid false memories. Journal of Experimental Psychology: Learning, Memory and Cognition, 31, 954-963.doi:10.1037/0278-7393.31.5.954

Light, L. L., Patterson, M. M., Chung, C., \& Healy, M. R. (2004). Effects of repetition and response deadline on associative recognition in young and older adults. Memory \& Cognition, 32, 11821193. doi:10.3758/BF03196891

Light, L. L., \& Singh, A. (1987). Implicit and explicit memory in young and older adults. Journal of Experimental Psychology: Learning, Memory and Cognition, 13, 531-541.

McCabe, D. P., Roediger III, H. L., McDaniel, M. A., \& Balota, D. A. (2009). Aging reduces veridical remembering but increases false remembering: Neuropsychological test correlates of remember-know judgements. Neuropsychologia, 47, 2164-2173. doi:10.1016/j. neuropsychologia.2008.11.025

Norman, K. A., \& Schacter, D. L. (1997). False recognition in younger and older adults: Exploring the characteristics of illusory memories. Memory \& Cognition, 25, 838-848. doi:10.3758/ BF03211328

Old, S. R., \& Naveh-Benjamin, M. (2008). Differential effects of age on item and associative measures of memory: A meta-analysis. Psychology and Aging, 23, 104-118. doi:10.1037/08827974.23.1.104 
Pitarque, A., Sales, A., Meléndez, J. C., \& Algarabel, S. (2015). Repetition increases false recollection in older people. Scandinavian Journal of Psychology, 56, 38-44. doi:10.1111/ sjop.2015.56.issue-1

Rhodes, M. G., Castel, A. D., \& Jacoby, L. L. (2008). Associative recognition of face pairs by younger and older adults: The role of familiarity- based processing. Psychology and Aging, 23, 239-249. doi:10.1037/0882-7974.23.2.239

Roediger III, H. L., Watson, J. M., McDermott, K. B., \& Gallo, D. A. (2001). Factors that determine false recall: A multiple regression analysis. Psychonomic Bulletin \& Review, 8, 385-407. doi:10.3758/ BF03196177

Rotello, C. M., \& Heit, E. (2000). Associative recognition: A case of recall-to-reject processing. Memory \& Cognition, 28, 907-922. doi:10.3758/BF03209339

Solé-Padullés, C., Bartrés-Faz, D., Junqué, C., Vendrell, P., Rami, L., Clemente, I., ... Molinuevo, J. L. (2009). Brain structure and function related to cognitive reserve variables in normal aging, mild cognitive impairment and Alzheimer's disease. Neurobiology of Aging, 30, 1114-1124. doi:10.1016/j.neurobiolaging.2007.10.008

Stern, Y. (2009). Cognitive reserve. Neuropsychologia, 47, 2015-2028. doi:10.1016/j. neuropsychologia.2009.03.004

Stern, Y., Habeck, C., Moeller, J., Scarmeas, N., Anderson, K. E., Hilton, H. J., ... Heertum, R. V. (2005). Brain networks associated with cognitive reserve in healthy young and old adults. Cerebral Cortex, 15, 394-402. doi:10.1093/cercor/bhh142

Valenzuela, M., \& Sachdev, P. (2006). Brain reserve and dementia: A systematic review. Psychological Medicine, 36, 441-454. doi:10.1017/S0033291705006264

Yonelinas, A. P. (2002). The nature of recollection and familiarity: A review of 30 years of research. Journal of Memory and Language, 46, 441-517. doi:10.1006/jmla.2002.2864

Zahodne, L. B., Glymour, M. M., Sparks, C., Bontempo, D., Dixon, R. A., MacDonald, S. W. S., \& Manly, J. J. (2011). Education does not slow cognitive decline with aging: 12-year evidence from the Victoria Longitudinal Study. Journal of the International Neuropsychological Society, 17, 10391046. doi:10.1017/S1355617711001044 